\title{
Distributed lightning monitoring: an affordable proposal
}

\author{
A. Luque $^{1}$ - F. Aguayo ${ }^{1}$ J. R. Lama ${ }^{1}$
}

Received: 28 December 2015 / Revised: 25 April 2016/Accepted: 9 May 2016 /

Published online: 19 May 2016

(C) The Author(s) 2016. This article is published with open access at Springerlink.com

\begin{abstract}
In theaters and the filmmaking industry, video streams, images, audio streams and scalar data are commonly used. In these fields, one of the most important magnitudes to be collected and controlled is the light intensity in different scene spots. So, it is extremely important to be able to deploy a network of light sensors which are usually integrated in a more general Wireless Multimedia Sensor Network (WMSN). If many light measurements have to be acquired, the simpler and cheaper the sensor, the more affordable the WMSN will be. In this paper we propose the use of a set of very cheap light sensors (photodiodes) and to spectrally and directionally correct their measurements using mathematical methods. A real testing of the proposed solution has been accomplished, obtaining quite accurate light measurements. Testing results are also presented throughout the paper.
\end{abstract}

Keywords Wireless Multimedia Sensor Networks $\cdot$ Light measurement $\cdot$ Light spectral correction $\cdot$ Light directional correction $\cdot$ Distributed systems

\section{Introduction}

There are many practical situations where distributed lighting monitoring systems are used for improving illuminance control, human health and comfort, industrial security or power efficiency. In those cases a common approach is to deploy a Sensor Network which can integrate, not only light information, but also some other magnitudes or pieces of information (sounds, images, pollution levels, temperature...). The communication of all these sensors are usually achieved through wireless connections creating what it is called a Wireless Multimedia Sensor Networks (WMSN).

The increasingly use of WMSN has been enabled by the availability, particularly in recent years, of sensors that are ever smaller, cheaper, and more intelligent. These sensors are usually equipped with wireless interfaces with which they can communicate with one another to build up a network. The

\section{A. Luque}

amalialuque@us.es

1 Departamento Ingeniería del Diseño, Universidad de Sevilla, Escuela Politécnica Superior - C/Virgen de África, 7, 41011 Sevilla, Spain 
design of a WMSN depends significantly on the application, and it must consider factors such as the environment, the application's design objectives, cost, hardware, and system constraints $[17,32]$.

One quite widespread application of this technology is street light control [25]. Some of these systems are based just on energy use monitoring [13], although most of them also incorporate light sensors to detect human presence (light needs) and illuminance level (light achieved) [20]. In these types of applications, several technical problems have to be addressed such as the routing through the WMSN [19] or the dependence and compensation of the sensor positions [40].

Another application where these technologies are extensively used is light control in smart buildings, usually also integrating much different information [12]. In regards to the topics covered in the paper, recent developments have tackled the energy cost reduction when used in the Real Time Pricing (RTP) framework of smart grids [14], the adaptive behavior of the system through self-calibrating and self-learning [39], and the sensor's cost concern when scaling the size of the network [8].

A further very promising field, although not yet exploited enough, is theaters and the filmmaking industry where deploying WMSN technologies could be very useful to integrate information provided for different classes of sensors integrating the simultaneous use of video streams, images, audio streams and scalar data. For this application, lighting control is extremely important $[2,3]$. Computerized control systems for lights in films and theaters are a well-established technology where even several commercial products are available [4, 9]. Lighting control on stages has also attracted the attention of recent research work which has addressed different aspects of the problem [18, 33, 36, 38].

However most of these current systems only provide for actuation and do not take advantage of sensor data to improve the control. It is important to know and use the live light information from light sensors deployed on the set. Real-time data accounts for how features, like light intensity and color temperature, change over time and deployments due to filament aging, supply voltage variation, changes in fixture position, color filters, etc. Without real-time measurements of light, it is time-consuming to maintain desired intensities of lights for certain areas across many venues and over long time periods. Light intensities can be measured accurately by currently commercially available handheld manual light meters [21, 35]. However, these devices have not been incorporated in systems supporting automatic light control and must be manually moved through different points in space. Cameras can provide only reflected light intensity, so we focus on incident light in order to have measurements that are independent of surfaces and materials.

The process of checking that adequate lighting conditions are met is a well-established task and there exist reliable measuring instruments (light meters) for a medium price. However the continuous monitoring of the adequacy of the light level requires distributed light sensors all through the stage and its subsequent integration into a control system, as it has been proposed in [29] for the entertainment and media production applications. Obviously, a better light monitoring and control is achieved where a higher spatial resolution is used, that is, where more light spots are measured.

Light sensing is an evolving field with new devices continuously being available. In these advances different features are being addressed like noise immunity [1], mechanical flexibility [22] or suitability to be integrated in WSN [5]. But in cases when the number of spots to be monitored increases, the cost of the sensors becomes one of the key factors.

A good light sensor device should include two important elements: a homogeneous directional response, whatever the direction in which the light is received from (flat directional sensitivity); and fitting the spectral response of the human eye. Most of the light sensors do not 
comply with these features and often require directional correction devices (usually superimposed lenses) and spectral compensation (optical filters and/or the combined use of different types of sensors). These correction mechanisms increase the price of each sensor.

In this work a different approach is proposed where it is shown that light measurement can be corrected both spectrally and directionally by numerical methods without requiring any additional hardware at each sensor. This allows low cost distributed systems with numerous spots of light measurements in a theater installation or film set. This paper describes the process and provides the necessary correction values for different lighting conditions. Similar solutions have been reported for calibration of wearable light exposure devices [10, 24] where only one light spot is needed and more sophisticated light sensors are used.

In section 2, a particular light sensor is selected and its performance measuring light intensity is derived. Section 3 addresses the problem of the sensor's spectral sensitivity, obtaining the analytical background for spectral correction. In section 4 the photodiode's behavior under different light direction is tackled, presenting the method for directionally correcting the sensor's measurements. The application of the proposed system is undertaken in section 5, where the main results are also described. Eventually, section 6 emphasizes the main paper's contributions and conclusions.

\section{The sensor}

For an easier understanding of the proposed method we will make our description, not only for an abstract sensor, but also applying the results to a specific device. For measuring light intensity we will use a photodiode OSRAM SFH 213 as the sensor device. We look for the relationship between light intensity and electric current in lighting conditions with different spectral compositions and various directional distributions. In a photodiode, the equation governing the behavior of the device [27] includes a term which is a function of the light intensity:

$$
i_{d}=I_{S}\left[e^{\frac{v_{d}}{n V_{T}}-1}\right]-I_{P}
$$

where $i_{d}$ is the current in the diode under a voltage $v_{d}$. The parameter $I_{s}$ is the reverse-bias saturation current. The parameter $V_{T}$ is the thermal voltage (a constant at a certain temperature). The parameter $n$ is the emission coefficient (in the range $1 \leq n \leq 2$ ). In (1) $\mathrm{I}_{\mathrm{P}}=K_{v} E_{v}$, where $E_{v}$ denotes the illuminance, i.e., the visible light power received per unit area in the diode, and $K_{v}$ is a constant for every photodiode.

The electric current in the photodiode when it is reverse biased $\left(v_{d}<0\right)$ is a good light intensity meter because the term

$$
I_{s}\left[e^{\frac{v_{d}}{n V_{T}}-1}\right]
$$

is negligible compared to $I_{p}$. In these cases

$$
\left|i_{d}\right| \approx I_{P}=K_{v} E_{v}
$$

In the case of the used photodiode it is possible to determine the proportionality constant from the values $\left(I_{R}=1 n A ; I_{p}=135 \mu A @ E_{v}=1000 l x\right)$ obtained in its data sheet [28]. Indeed, we start by determining the value of $I_{s}$.

$$
\left.I_{R} \equiv i_{d}\right|_{v_{d}=-\infty ; E_{v}=0}=I_{S}\left[e^{\frac{-\infty}{n V_{T}}-1}\right]-K_{v} \cdot 0=-I_{S} \rightarrow I_{S}=-I_{R}=1 n A
$$


Additionally

$$
K_{v}=\frac{I_{P}+I_{s}}{-E_{v}}=\frac{-135 \mu A+1 n A}{-1000 l x}=135 \frac{n A}{l x}
$$

These measurements are made with a standard type A light (corresponding to an incandescent lamp) and in a front direction with respect the photodiode. In the following sections the relationship between light intensity and electric current will become clear, in cases of lighting with different spectral compositions and different directional distributions.

\section{Spectral sensitivity}

The radiant flux $\Phi_{\mathrm{e}}$ is defined as the total power emitted by a light source or received by a light sensor. On the other hand, the luminous flux $\Phi_{\mathrm{v}}$ is defined as the visible power emitted by the source or received by the sensor. Illuminance $E_{v}$ (lux) is also defined as the visible power received per unit area. For sensor with area $A$

$$
E_{v}=\frac{\Phi_{\mathrm{v}}}{A}
$$

The spectral response of the human eye is not equal for all wavelengths (colors) of light. Although it can vary slightly from one individual to another and depends on lighting conditions, the photopic $\mathrm{V}_{\lambda}$ (in daylight conditions) response is standardized in [16]. If the spectral density of the radiant flux emitted by certain type A light source is denoted as $\Phi_{\mathrm{e} \lambda}$, the spectral density $\Phi_{\mathrm{v} \lambda}$ of luminous flux (perceived by human eye) will be

$$
\Phi_{\mathrm{v} \lambda}=\Phi_{\mathrm{e} \lambda} \mathrm{V}_{\lambda}
$$

If a sensor with spectral sensitivity $S_{\lambda}$ is used to measure visible light, the spectral density of luminous flux measured by the sensor will be

$$
\Phi_{\mathrm{s} \lambda}=\Phi_{\mathrm{e} \lambda} S_{\lambda}
$$

The function of the spectral sensitivity of the sensor can be determined from its data sheet. A standard light source is one that emits light with a certain (standardized) spectral distribution. For example, a standard type A light [15] is the light corresponding to a lamp with a tungsten filament at $2856^{\circ} \mathrm{K}$ of temperature corresponding to a standardized spectral distribution $\Phi_{\mathrm{eA} \lambda \mathrm{n}}$. Any type A light source, will have a distribution $\Phi_{\mathrm{eA \lambda}}$ with the same shape as the standard one, but multiplied by a constant $L_{A}$, that is,

$$
\Phi_{\mathrm{eA \lambda}}=L_{A} \Phi_{\mathrm{eA} \lambda \mathrm{n}}
$$

Other standard light sources of interest are type D lights [15], corresponding to natural daylight, and the family of type F lights [6] corresponding to different types of fluorescent lights (F1 to F12). Suppose we have a standard type A light source with a spectral distribution of radiant flux given by (9). The radiant flux emitted by the type A light source will be 


$$
\Phi_{\mathrm{eA}}=\int_{0}^{\infty} \Phi_{\mathrm{eA} \lambda} d \lambda=L_{A} \int_{0}^{\infty} \Phi_{\mathrm{eA} \lambda \mathrm{n}} d \lambda=L_{A} \Phi_{\mathrm{eAn}}
$$

an expression in which

$$
\Phi_{\mathrm{eAn}} \equiv \int_{0}^{\infty} \Phi_{\mathrm{eA} \lambda \mathrm{n}} d \lambda=4.4396 \cdot 10^{5} \text { watts }
$$

value obtained integrating the curve of the spectral distribution of radiant flux of type A light. Analogously, considering the luminous flux (perceived by the human eye), $\Phi_{\mathrm{vA}}=L_{A} \Phi_{\mathrm{vAn}}$, where

$$
\Phi_{\mathrm{vAn}} \equiv \int_{0}^{\infty} \Phi_{\mathrm{vA} \lambda \mathrm{n}} d \lambda=1.0788 \cdot 10^{4} \mathrm{~lm}
$$

And considering the flux perceived by the sensor, $\Phi_{\mathrm{sA}}=L_{A} \Phi_{\mathrm{sAn}}$, where

$$
\Phi_{\mathrm{sAn}} \equiv \int_{0}^{\infty} \Phi_{\mathrm{sA \lambda n}} d \lambda=9.7898 \cdot 10^{4} \text { watts. }
$$

Values of $\Phi_{v A n}$ and $\Phi_{S A n}$ are obtained integrating the corresponding curves of the spectral distribution of a type A light and its perception by the eye and the sensor. It is called normalized spectral sensitivity, in this case for a type A light, the value

$$
\eta_{\mathrm{A}} \equiv \frac{\Phi_{\mathrm{sA}}}{\Phi_{\mathrm{vA}}}
$$

From the above expressions it is possible to develop and get that

$$
\eta_{\mathrm{A}}=\frac{\Phi_{\mathrm{sAn}}}{\Phi_{\mathrm{vAn}}}
$$

In the case of our sensor, normalized spectral sensitivity to type A light value is

$$
\eta_{\mathrm{A}}=\frac{\Phi_{\mathrm{sAn}}}{\Phi_{\mathrm{vAn}}}=9.07
$$

From the above expressions it is possible to deduct that

$$
\Phi_{\mathrm{vA}}=\frac{\Phi_{\mathrm{sA}}}{\eta_{\mathrm{A}}} .
$$

From (3) we can see that the current supplied by the photodiode in the case of excitation with light A will be

$$
I_{P A}=K_{v} E_{v A}=\frac{K_{v}}{\eta_{\mathrm{A}}} \cdot \frac{\Phi_{\mathrm{sA}}}{A}
$$

Calling $K_{s} \equiv \frac{K_{v}}{\eta_{\mathrm{A}}}$, we can substitute in the above expression and obtain finally that

$$
I_{P A}=K_{s} \frac{\Phi_{\mathrm{sA}}}{A}
$$

In the case of our sensor, the value of the constant $K_{S}$ is

$$
K_{s} \equiv \frac{K_{v}}{\eta_{\mathrm{A}}}=14.88 \frac{n A}{\frac{\text { watt }}{m^{2}}}
$$


Suppose now that we have a generic light source with a spectral distribution of radiant flux given by $\Phi_{\mathrm{e} \lambda}$. The luminous flux (perceived by the human eye) will be

$$
\Phi_{\mathrm{v}}=\int_{0}^{\infty} \Phi_{\mathrm{e} \lambda} V_{\lambda} d \lambda
$$

Meanwhile the flux perceived by the sensor will be

$$
\Phi_{\mathrm{s}}=\int_{0}^{\infty} \Phi_{\mathrm{e} \lambda} S_{\lambda} d \lambda
$$

It is called normalized spectral sensitivity for any type of light

$$
\eta \equiv \frac{\Phi_{\mathrm{s}}}{\Phi_{\mathrm{v}}}
$$

The current supplied by the photodiode in the case of a generic excitation light will be

$$
I_{P}=K_{s} \frac{\Phi_{\mathrm{s}}}{A}=K_{s} \frac{\eta \Phi_{\mathrm{v}}}{A} .
$$

The relationship between the current supplied by the photodiode when excited with generic light and when excited with type A light is called spectral correction factor $\sigma$, assuming that both are illuminated with the same luminous flux. This relationship can be expressed as

$$
\sigma \equiv \frac{I_{P}}{I_{P A}}=\frac{K_{s} \frac{\eta \Phi_{\mathrm{v}}}{A}}{K_{v} \frac{\eta_{\mathrm{A}} \Phi_{\mathrm{v}}}{A}}=\frac{\eta}{\eta_{\mathrm{A}}}
$$

This value, properly calculated, can be used to determine the current provided by the photodiode

$$
I_{P}=\sigma I_{P A}=\sigma K_{v} E_{v}
$$

Applying these criteria to the sensor illuminated with a type D (daylight) light, we find that its normalized spectral sensitivity is

$$
\eta_{\mathrm{D}} \equiv \frac{\Phi_{\mathrm{sD}}}{\Phi_{\mathrm{vD}}}=\frac{\int_{0}^{\infty} \Phi_{\mathrm{eD} \lambda} S_{\lambda} d \lambda}{\int_{0}^{\infty} \Phi_{\mathrm{eD} \lambda} V_{\lambda} d \lambda}=2.72
$$

This value is obtained integrating the corresponding curves of the spectral distribution of a $\mathrm{D}$ light and its perception by the eye and the sensor. The spectral correction will therefore be

$$
\sigma_{\mathrm{D}}=\frac{\eta_{\mathrm{D}}}{\eta_{\mathrm{A}}}=0.30
$$

Similarly, considering the case of a light sensor illuminated by a type F1 light (fluorescent light), we find that its normalized spectral sensitivity is

$$
\eta_{\mathrm{F}} \equiv \frac{\Phi_{\mathrm{SF}}}{\Phi_{\mathrm{vF}}}=\frac{\int_{0}^{\infty} \Phi_{\mathrm{eF} \lambda} S_{\lambda} d \lambda}{\int_{0}^{\infty} \Phi_{\mathrm{eF} \lambda} V_{\lambda} d \lambda}=1.016
$$


This value is obtained integrating the corresponding curves of the spectral distribution of type F1 light and its perception by the eye and the sensor. The spectral correction will therefore be

$$
\sigma_{\mathrm{F}}=\frac{\eta_{\mathrm{F}}}{\eta_{\mathrm{A}}}=0.112
$$

For other illuminations of type $\mathrm{F}$ family lights, the results for the normalized spectral sensitivity and the spectral correction are shown in the following table.

\begin{tabular}{lllllllllllll}
\hline & $F_{1}$ & $F_{2}$ & $F_{3}$ & $F_{4}$ & $F_{5}$ & $F_{6}$ & $F_{7}$ & $F_{8}$ & $F_{9}$ & $F_{10}$ & $F_{11}$ & $F_{12}$ \\
$\eta_{F}$ & 1.016 & 0.966 & 0.956 & 0.972 & 0.985 & 0.927 & 1.255 & 1.412 & 1.363 & 0.988 & 1.002 & 1.030 \\
$\sigma_{F}$ & 0.112 & 0.107 & 0.106 & 0.107 & 0.109 & 0.102 & 0.138 & 0.156 & 0.150 & 0.109 & 0.111 & 0.114 \\
\hline
\end{tabular}

\section{Directional sensitivity}

In the data sheet of the sensor which has allowed us to obtain the relationship between light intensity and electric current two considerations are made: that light is of type A and falling on the photodiode in a front direction. The first of these issues has been addressed in the previous section. In this section the consequences of light falling on the sensor not frontally will be discussed.

Suppose that a sensor is illuminated with a light radiation of a certain source. $M_{v}$ denotes the illuminance (lumen $/ \mathrm{m}^{2}$ ), that is, the light power (visible) per unit area emitted by the source. $\Phi_{\mathrm{v}}$ denoted the luminous flux (visible) received by the sensor. For a sensor with an area $A_{0}$ subjected to a uniform perpendicular illuminance, the relationship between the two magnitudes is

$$
\Phi_{\mathrm{v} 0}=M_{v} A_{0}
$$

For the case in which the sensor is subjected to a uniform lighting luminous emittance inclined at an angle $\varphi$ relative to the perpendicular, the relationship becomes [23]

$$
\Phi_{\mathrm{v} \varphi}=M_{v} A_{0} \cos \varphi=\Phi_{\mathrm{v} 0} \cos \varphi
$$

It is called directional sensitivity of the sensor the expression

In this case

$$
\mathrm{S}_{\varphi}=\mathrm{S}(\varphi) \equiv \frac{\Phi_{\mathrm{v} \varphi}}{\Phi_{\mathrm{v} 0}}
$$

$$
\mathrm{S}_{\varphi}=\cos \varphi
$$

known as the Lambert's cosine law [31]. With the above definitions we can write

$$
\Phi_{\mathrm{v} \varphi}=S_{\varphi} \Phi_{\mathrm{v} 0}
$$

It can be shown similarly (the complete demonstration is omitted for space reasons) that expressions allowing one to calculate the correction factor with any lighting conditions (Fig. 1) and with a sensor (blue segment in the figure) inclined an angle $\theta$, are the following:

$$
\psi(\theta)=\frac{M_{v a}(\theta)}{M_{v}(\theta)}
$$


Where

$$
\begin{gathered}
M_{v}(\theta)=\int_{0}^{\frac{\pi}{2}}\left[\int_{-\pi}^{+\pi} H_{v}(\alpha, \varepsilon) d \alpha^{\prime}\right] d \varepsilon^{\prime} \\
M_{v a}(\theta)=\int_{0}^{\frac{\pi}{2}}\left[\int_{-\pi}^{+\pi} H_{v}(\alpha, \varepsilon) S(\beta) d \alpha^{\prime}\right] d \varepsilon^{\prime}
\end{gathered}
$$

and $H_{v}(\alpha, \varepsilon)$ is the density of emittance for a certain azimuth $\alpha$ and elevation $\varepsilon$ into the room's reference system $(X, Y, Z)$ defined as

$$
H_{v}(\alpha, \varepsilon) \equiv \frac{d E_{v}}{d \alpha d \varepsilon}
$$

The variable $\beta$ is the angle between the sensor plane and the light.

In these expressions a change of variables is needed to express all the elements as a function of the azimuth $\alpha^{\prime}$ and elevation $\varepsilon^{\prime}$ into the sensor's reference system $\left(X^{\prime}, Y^{\prime}, Z^{\prime}\right)$. The change of variables is, after some basic trigonometric operations,

$$
\begin{gathered}
\alpha=\operatorname{arctg} \frac{\cos \varepsilon^{\prime} \operatorname{sen} \alpha^{\prime}}{\cos \varepsilon^{\prime} \cos \alpha^{\prime} \cos \theta+\operatorname{sen} \varepsilon^{\prime} \operatorname{sen} \theta} \\
\varepsilon=\operatorname{arctg} \frac{-\cos \varepsilon^{\prime} \cos \alpha^{\prime} \operatorname{sen} \theta+\operatorname{sen} \varepsilon^{\prime} \cos \theta}{\sqrt{\left(\cos \varepsilon^{\prime} \cos \alpha^{\prime} \cos \theta+\operatorname{sen} \varepsilon^{\prime} \operatorname{sen} \theta\right)^{2}+\left(\cos \varepsilon^{\prime} \operatorname{sen} \alpha^{\prime}\right)^{2}}} \\
\beta=\frac{\pi}{2}-\varepsilon^{\prime}
\end{gathered}
$$

\section{Application and results}

Results of previous sections have been applied to a specific situation in testing conditions. The measurements were performed in a hall with all the (fluorescent) lights on, and doors and

Fig. 1 Room's and sensor's reference systems

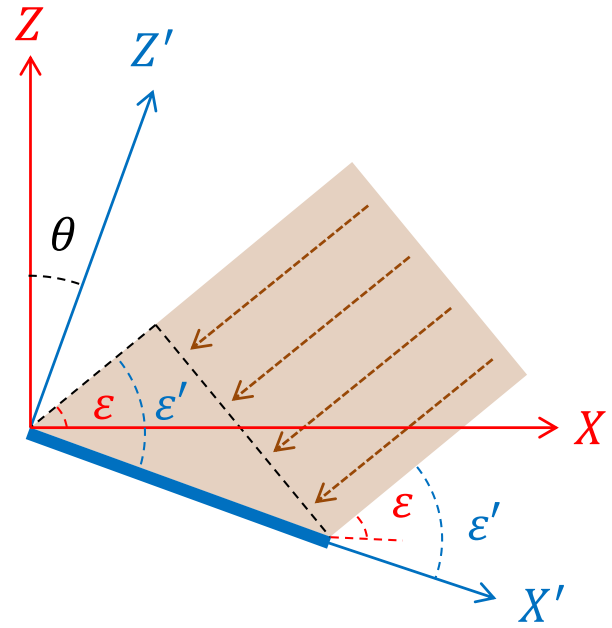


Fig. 2 Measurements of the photodiode current for various inclinations $(\alpha=0)$

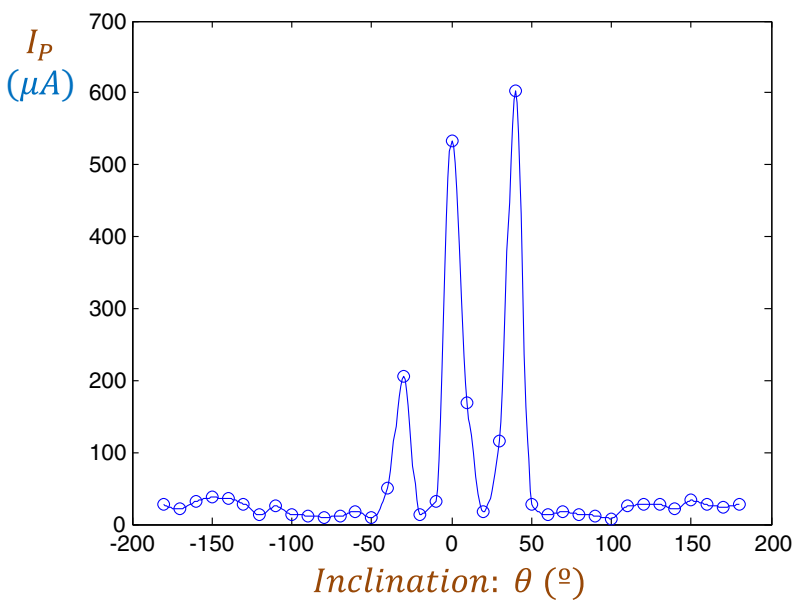

windows closed. The photodiode under study has been used as light sensor. It was introduced in a long, narrow opaque cylinder that gives an angular resolution of approximately $\phi=3^{\circ}\left( \pm 1.5^{\circ}\right.$. With this reduced opening angle, the sensitivity of the photodiode can be considered constant. The device was placed just below a fluorescent lamp, with two additional lamps seen by the sensor with inclinations of $30^{\circ}$ to the left and $40^{\circ}$ to the right. The sensor configured in that way had been oriented on a set of angles of azimuth and elevation, covering the entire space. The value provided by the sensor at each position was an electric current. The measurements obtained for an azimuth $\alpha=0$ and for a variable inclination $\theta$ are shown in Fig. 2. The three peaks in the graphic correspond to the three closest lamps on the ceiling above the sensor.

Similarly, Fig. 3 shows the measurements of electric current obtained by the sensor depending on the elevation $\varepsilon$ for different values of azimuth $(\alpha)$.

Figure 4 performs a 3D representation of the measurements obtained by the sensor for the entire hall space. If this information about the light spatial distribution is not available, it can be estimated for the measurements of a grid of sensors [7, 26, 34].

Fig. 3 Measurements of the photodiode current for various elevations (variable azimuth)

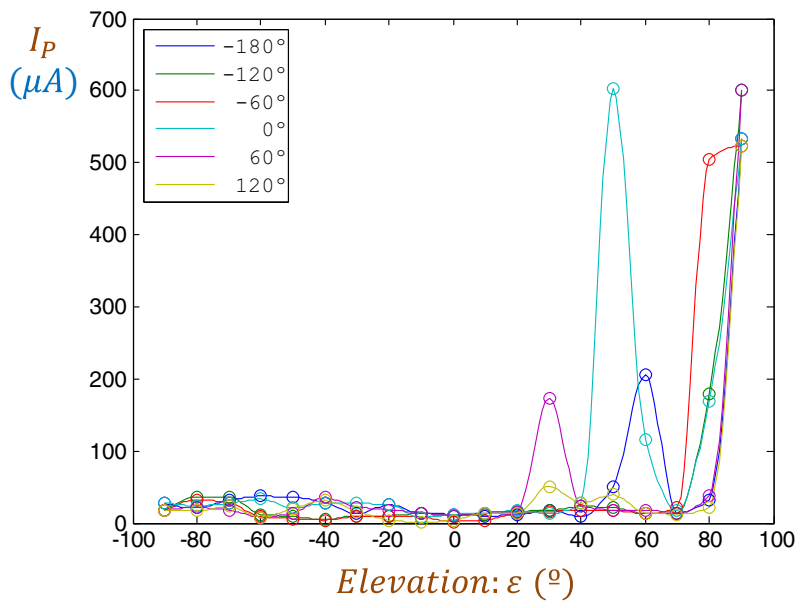


Fig. 4 Measurements obtained by the sensor (3D representation)

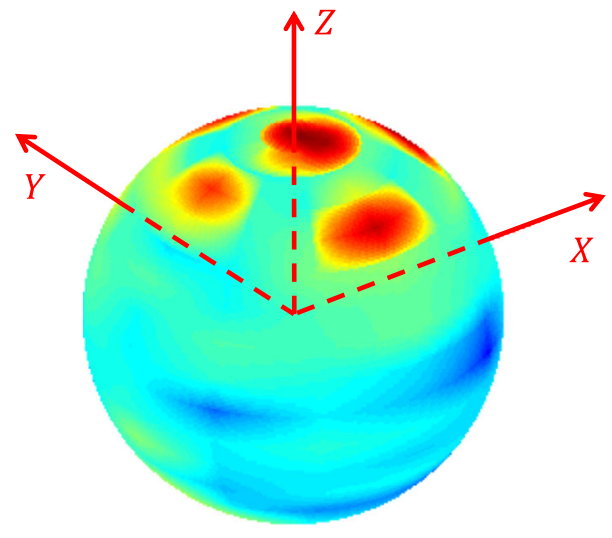

For a certain elevation $\varepsilon$ and azimuth $\alpha$, the relationship between measures of electric current and lighting intensity can be derived from (15) and (28)

$$
H_{v}(\alpha, \varepsilon)=\frac{I_{P}(\alpha, \varepsilon)}{\sigma K_{v} \phi^{2}}
$$

expression in which $\phi$ represents a small opening angle on the measure of the sensor where $H_{v}$ can be considered constant. In order to experimentally determine the values of the spectral correction $(\sigma)$ and directional $(\psi)$ factors, a reliable measure of the illumination values for each angle is needed. For this purpose we use a commercial PCE-172 luxmeter [30]. This device incorporates the cosine correction (uniform directional sensitivity); and it has a spectral response adjusted to the normalized photopic response. With this luxmeter, lighting for different angles is determined. Substituting (32) in (26), it can be written that

$$
M_{v}(\theta)=\frac{1}{\sigma K_{v} \phi^{2}} \int_{0}^{\frac{\pi}{2}}\left[\int_{-\pi}^{+\pi} I_{P}(\alpha, \varepsilon) d \alpha^{\prime}\right] d \varepsilon^{\prime}
$$

expression in which the values of $I_{P}(\alpha, \varepsilon)$ (experimentally determined) are known; the value of $K_{v}$ (from the data sheet of the sensor; see previous sections); and also the value of $\phi$ (opening angle), geometrically determined from the dimensions of the cylinder in which the photodiode is introduced. The only unknown value is the coefficient of spectral correction $\sigma$. The value of the emittance without spectral correction, denoted $\tilde{M}_{v}$

$$
\begin{gathered}
\tilde{M}_{v}(\theta)=\frac{1}{K_{v} \phi^{2}} \int_{0}^{\frac{\pi}{2}}\left[\int_{-\pi}^{+\pi} I_{P}(\alpha, \varepsilon) d \alpha^{\prime}\right] d \varepsilon^{\prime} \\
M_{v}(\theta)=\frac{1}{\sigma} \tilde{M}_{v}(\theta)
\end{gathered}
$$

In Fig. 5 both the experimental results obtained using the luxmeter are presented, and the theoretical values of $M_{v}(\theta)$ derived from (35) for different values of $\sigma$.

As shown in the graph, a spectral correction factor value $\sigma=0.17$ achieves a good fit between experimental and theoretical values. However, such adjustment can be optimized by numerical methods. Indeed, let's denominate $E_{v}\left(\theta_{i}\right)$ the experimental illuminance measured by the light meter for an inclination $\theta_{i}$; and let's denominate $M_{v}\left(\theta_{i}\right)$ the theoretically calculated 
Fig. 5 Illuminance depending on the inclination: experimental and theoretical values

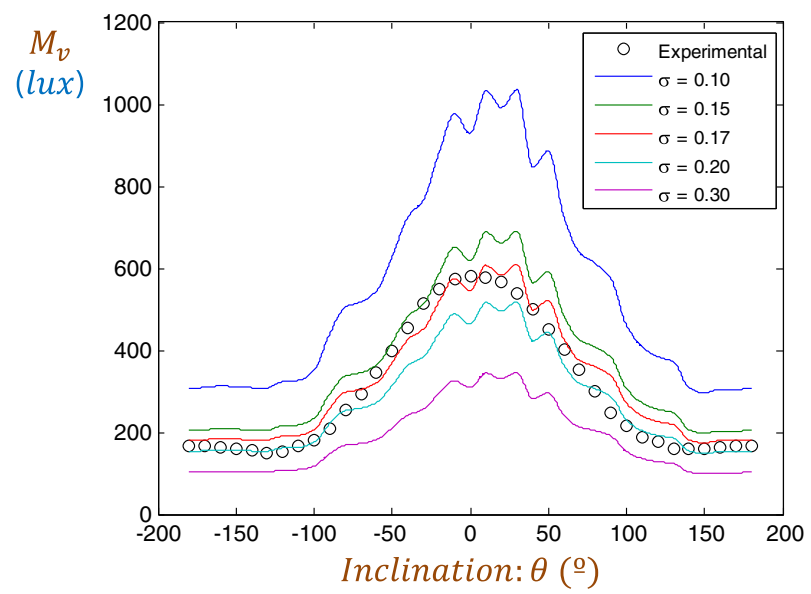

emittance for this same angle $\theta_{i}$, with $N$ inclinations angles being considered. The mean square error obtained for a given value of the spectral correction factor will be

$$
\begin{gathered}
\varepsilon_{c m}=\frac{1}{N} \sum_{i=1}^{N}\left[M_{v}\left(\theta_{i}\right)-E_{v}\left(\theta_{i}\right)\right]^{2} \\
\varepsilon_{c m}=\frac{1}{N} \sum_{i=1}^{N}\left[\frac{1}{\sigma} \tilde{M}_{v}\left(\theta_{i}\right)-E_{v}\left(\theta_{i}\right)\right]^{2}
\end{gathered}
$$

Minimizing the mean square error MSE, the optimum spectral correction factor is obtained, which in our case is $\sigma=0.17813$. Figure 6 shows the fit between the experimental and theoretical values with the optimal $\sigma$. If we assume both a $5 \%$ error in inclination and also in illuminance measures, every experimental dot stays inside the error bands depicted in the figure.

The obtained spectral correction value is close to the range corresponding to type $\mathrm{F}$ luminaires $(0.102 \leq \sigma \leq 0.156)$. The difference may be explained, in addition to experimental error, for the

Fig. 6 Illuminance depending on the inclination: optimal values

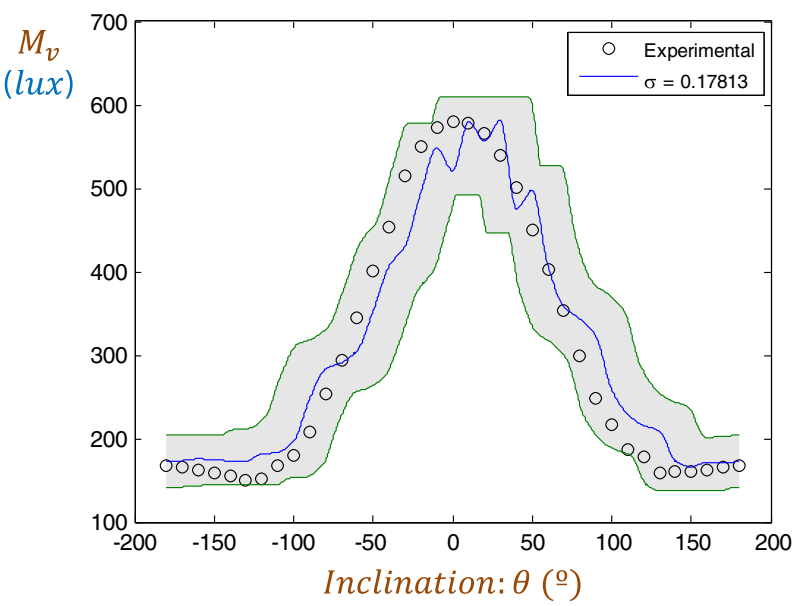


Fig. 7 Experimental and theoretical values of the illuminance (emittance) for both the photodiode and for the luxmeter

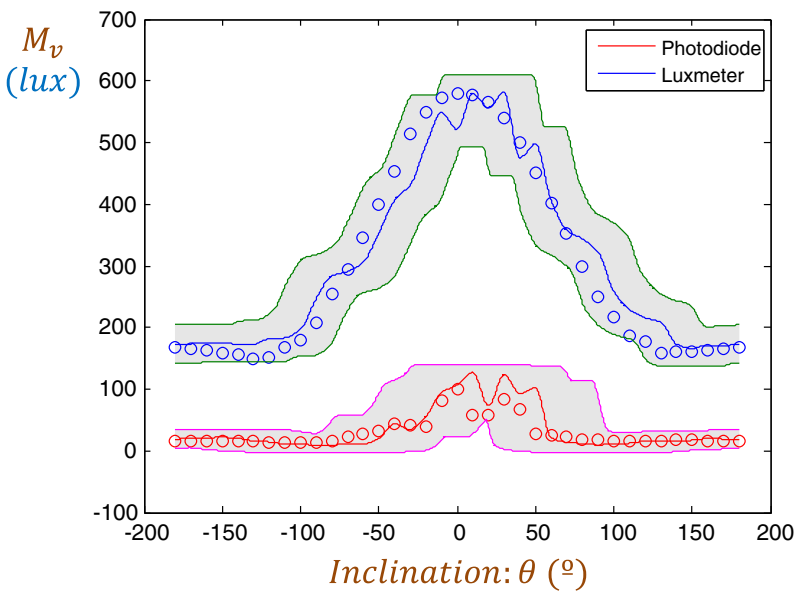

incidence on the sensor of light reflected by the room (not directly from the luminaire) that obviously alters the spectrum of light and therefore the spectral correction factor.

To determine the value of the directional correction factor $\psi$ the expression (25) is applied, where the value of $M_{v}(\theta)$ is given by (33), and the value of $M_{v a}(\theta)$ is derived substituting (32) in (27),

$$
M_{v a}(\theta)=\frac{1}{\sigma K_{v} \phi^{2}} \int_{0}^{\frac{\pi}{2}}\left[\int_{-\pi}^{+\pi} I_{P}(\alpha, \varepsilon) S(\beta) d \alpha^{\prime}\right] d \varepsilon^{\prime}
$$

expression in which we know every value. Figure 7 depicts the experimental and theoretical values of the illuminance (emittance) for both the photodiode $\left(M_{v a}\right)$ and for the luxmeter $\left(M_{v}\right)$. In the photodiode case a $10 \%$ error band in inclination and also in illuminance measures is depicted.

The quotient between the above two curves (25) is just the directional correction factor $\psi$. As expected, its value depends on the orientation $(\theta)$ as it is shown in Fig. 8.

For the final directional correction of the illuminance measurement the real inclination of the sensor has to be considered. The spectrally and directionally corrected photodiode

Fig. 8 Directional correction factor

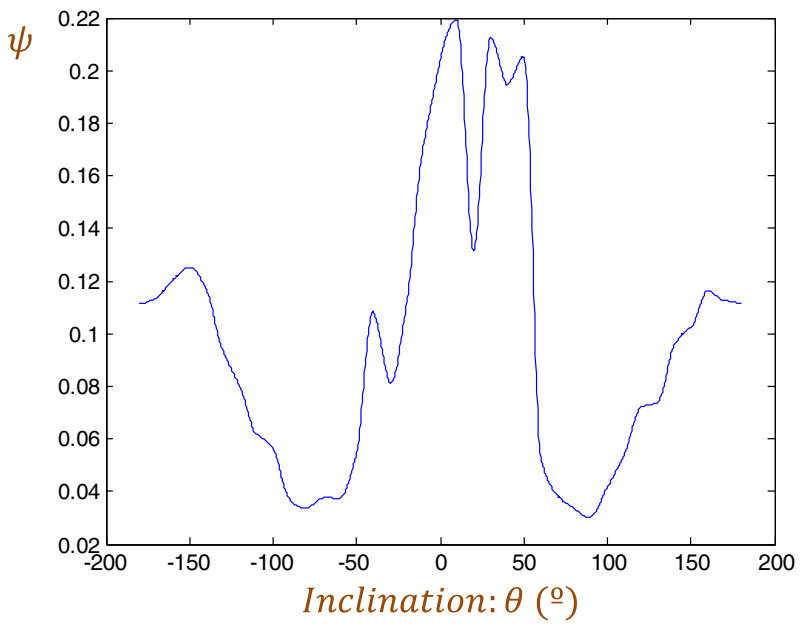


Fig. 9 Photodiode and luxmeter measurement

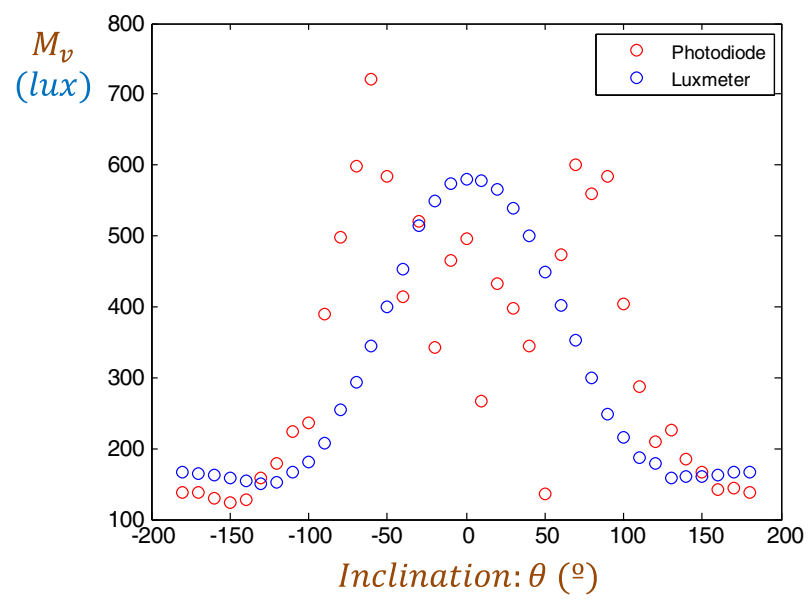

measurements are depicted with red dots in Fig. 9. With blue dots are represented the measurement obtained by a calibrated luxmeter.

As can be seen the error in the photodiode measurement also depends on inclination. In Fig. 10 this error is shown. The maximum error for any inclination is $65 \%$. For inclinations covering direct (or almost direct) lights, the errors are about $20 \%$. And for the indirect lights areas the errors are below $10 \%$. These results are similar to the $57 \%$ maximum error reported for an RGB photosensor [10], and much better results than the $152 \%$ error stated for a threecolor sensors equipped device [24]. The proposed method equals or supersedes previously reported research while using less complex (and cheaper) sensors.

Although having a $65 \%$ maximum error in the illuminance is not a good figure for laboratory measurements, it could be acceptable for applications where the goal is to control the light subjective perception, like in stage lighting. In fact, the relationship between illuminance level and brightness (subjective perception) is logarithmic [37], following Fechner's Law [11]. The error of the proposed method in brightness terms is about a $10 \%$ or below for most inclinations, and never higher than $20 \%$ (Fig. 11).

Fig. 10 Illuminance measurement error

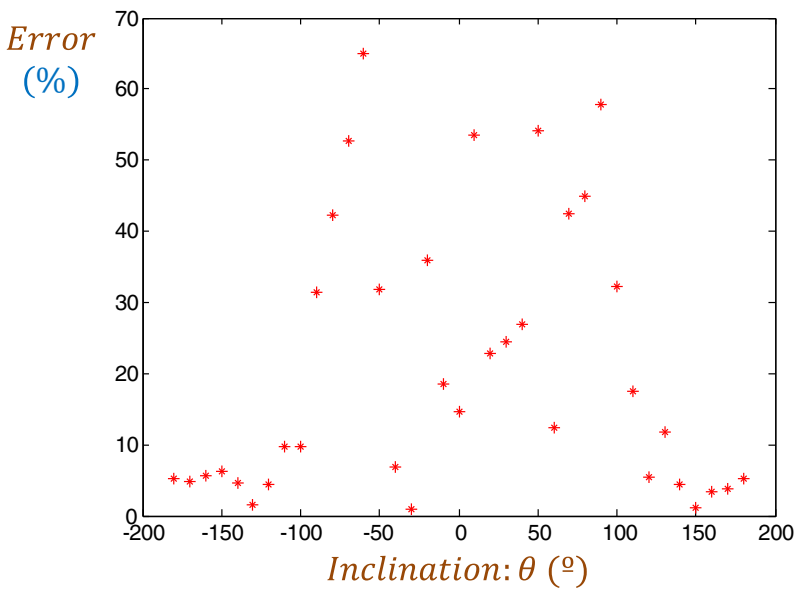


Fig. 11 Histogram of brightness error

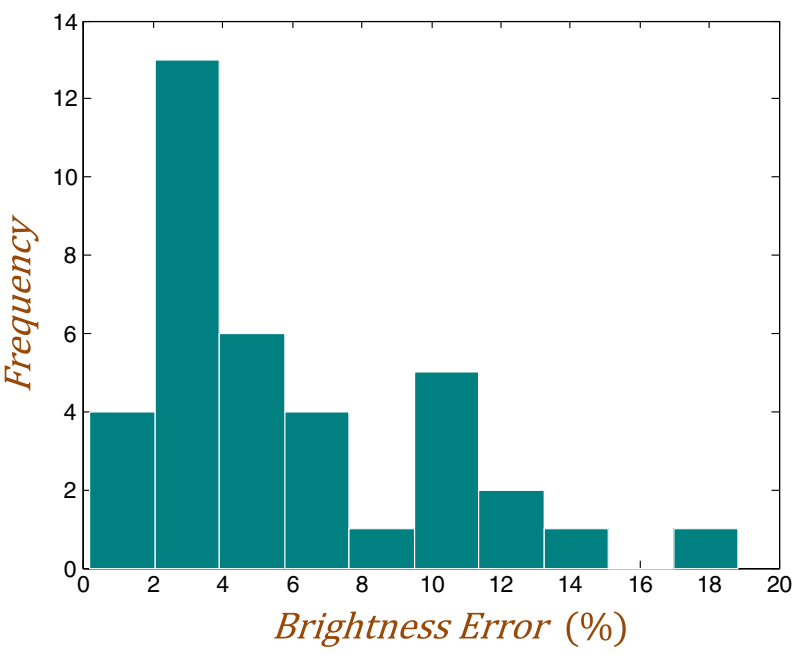

\section{Conclusions}

From the theoretical and experimental work described in the previous sections, it follows that it is possible to use a simple (and cheap) sensor (photodiode) as a light meter capable of providing quite accurate measurements of the illuminance and brightness. Low cost sensors, as the one proposed, require spectral and directional corrections which have been derived throughout the paper. The error analysis has demonstrated that our approach equals or supersedes results obtained in previous research, despite the fact that we use much simpler sensors.

This affordable approach permits the integration of a high number of light sensors in a more general WMSN. It has been shown that its use is possible in stage light monitoring building up Wireless Multimedia Sensor Networks. The use of this technology in theaters and filmmaking industry is proposed, integrating many spots light measurements with other multimedia information (video, images, audio and other scalar data).

Acknowledgments This work has been supported by the Telefonica Chair "Intelligence in Networks" of the University of Seville (Spain).

Open Access This article is distributed under the terms of the Creative Commons Attribution 4.0 International License (http://creativecommons.org/licenses/by/4.0/), which permits unrestricted use, distribution, and reproduction in any medium, provided you give appropriate credit to the original author(s) and the source, provide a link to the Creative Commons license, and indicate if changes were made.

\section{References}

1. Adamo G, Agrò D, Stivala S, Parisi A, Giaconia C, Busacca AC, Fallica G (2014). SNR measurements of silicon photomultipliers in the continuous wave regime. In: SPIE OPTO. International Society for Optics and Photonics, p 899019-899019

2. Box H (2013) Set lighting technician's handbook: film lighting equipment, practice, and electrical distribution. Taylor \& Francis

3. Cadena R (2012) Automated lighting: the art and science of moving light in theatre, live performance, and entertainment 2nd Edition. Taylor \& Francis 
4. Cast. URL: http://www.cast-soft.com/

5. Chiang CT (2014) Design of a CMOS monolithic digitized light detector with noise insensitivity for light monitoring applications. IEEE Sens J 14(8):2537-2545

6. CIE Technical Report: Colorimetry. Publication 15:2004 (3rd ed.)

7. DiBernardo E, Pirjanian P (2014) Methods and apparatus for position estimation using reflected light sources. U.S. Patent No. 8,780,342. Washington: U.S. Patent and Trademark Office

8. Dibley M, Li H, Rezgui Y, Miles J (2012) Cost effective and scalable sensor network for intelligent building monitoring. Int J Innov Comput Inf Control 8(12):8415-8433

9. Electronic Theatre Controls. URL: http:/www.etcconnect.com

10. Figueiro MG, Hamner R, Bierman A, Rea MS (2013) Comparisons of three practical field devices used to measure personal light exposures and activity levels. Light Res Technol 45(4):421-434

11. Heidelberger M (2004) Nature from within: Gustav Theodor Fechner and his psychophysical worldview. University of Pittsburgh Press

12. Higuera J, Hertog W, Perálvarez M, Carreras J (2014) Hybrid smart lighting and climate control system for buildings. In: Future Intelligent Cities, IET Conference on. IET, p 1-5

13. Howell DW, Vinson MW, Blevins FO, Tamagni AJ Jr, Campbell ML (2009) U.S. Patent No. 7,571,063. Patent and Trademark Office, Washington

14. Im KM, Lim JH (2015) Lighting control system based on the RTP of smart grid in WSN. Int J Distrib Sens Netwr

15. ISO 10526:1999/CIE S005/E-1998: CIE standard illuminants for colorimetry

16. ISO 23539:2005 (CIE S010/E:2004) Photometry - The CIE system of physical photometry

17. Iyengar SS, Brooks RR (Eds.) (2012) Distributed sensor networks: sensor networking and applications. CRC press

18. Jiang W, Jiang Y, Ren H (2010) Analysis and prospect of control system for stage lighting. In: Image and Signal Processing (CISP), 2010 3rd International Congress on, Vol. 8. IEEE, p 3923-3929

19. Jing C, Ren L, Gu D (2010) Geographical routing for WSN of street lighting monitoring and control system. In: Computer Design and Applications (ICCDA), 2010 International Conference on, Vol. 3. IEEE, p V3-235

20. Karthikeyan M, Saravanan V, Vijayakumar S (2014) Cloud based automatic street light monitoring system. In: Green Computing Communication and Electrical Engineering (ICGCCEE), 2014 International Conference on. IEEE, p 1-6

21. Konica Minolta T10A. URL: http://sensing.konicaminolta.asia/products/t-10a-illuminance-meter/

22. Maiellaro G, Ragonese E, Gwoziecki R, Jacobs S, Marjanovic N, Chrapa M, .. \& Palmisano G (2014) Ambient light organic sensor in a printed complementary organic tft technology on flexible plastic foil. IEEE Trans Circ Syst Regul Pap 61(4): 1036-1043

23. Malacara D (2001) Handbook of optical engineering. CRC Press

24. Markvart J, Hansen ÅM, Christoffersen J (2015) Comparison and correction of the light sensor output from 48 wearable light exposure devices by using a side-by-side field calibration method. LEUKOS 11(3):155-171

25. Mendalka M, Gadaj M, Kulas L, Nyka K (2010) WSN for intelligent street lighting system. In: Information technology (ICIT), 2010 2nd international conference on. IEEE, p 99-100

26. Miki M, Yoshida K, Hirano Y, Ikegami H (2013) Estimation of illuminance sensor positions and improvement of energy efficiency in the distributed control lighting system. In: Applied Computational Intelligence and Informatics (SACI), 2013 I.E. 8th International Symposium on. IEEE, p 137-142

27. Neamen DA (2007) Microelectronics: circuit analysis and design 3rd edition. McGraw-Hill

28. OSRAM Opto Semiconductors (2014) SFH 213 Datasheet. Retrieved from http://www.osram-os.com

29. Park H, Burke J, Srivastava MB (2007). Design and implementation of a wireless sensor network for intelligent light control. In: Proceedings of the 6th international conference on Information processing in sensor networks. ACM, p 370-379

30. PCE Instruments. PCE-172 light meter. URL: https://www.pce-instruments.com

31. Pedrotti FL, Pedrotti LM, Pedrotti LS (2013) Introduction to optics (3rd Edition). Pearson

32. Rawat P, Singh KD, Chaouchi H, Bonnin JM (2014) Wireless sensor networks: a survey on recent developments and potential synergies. J Supercomput 68(1):1-48

33. Ren H, Zhou K, Li Z, Wang HQ (2011) Research and realization of new stage lighting control system. In: Computational Sciences and Optimization (CSO), 2011 Fourth International Joint Conference on. IEEE, p 777-779

34. Schell S, Witman R, Brown J, Kerekes T (2005) Sensing device and method for measuring position and orientation relative to multiple light sources. U.S. Patent Application No. 11/090,405. Washington: U.S. Patent and Trademark Office

35. Sekonic i-346 Illuminometer. URL: http:/www.sekonic.com/products/i-346/overview.aspx

36. Shilei B, Wei J, Yujian J, Jingjing Z (2011) Research on Automatic Networking of Stage Lighting Devices and Its Key Technologies. In: Intelligent Computation Technology and Automation (ICICTA), 2011 International Conference on, Vol. 2. IEEE, p 661-664

37. Skalicky SE (2016) Luminance range for vision. In: Ocular and visual physiology. Springer Singapore, p 299-312 
38. Tian L, Du H, Gao C, Tang L, Xu Y (2013) The Development Condition of Lighting Control System on Stage. In: 2013 Sixth International Symposium on Computational Intelligence and Design

39. Wang Y, Dasgupta P (2015) Designing an adaptive lighting control system for smart buildings and homes. In: Networking, Sensing and Control (ICNSC), 2015 I.E. 12th International Conference on. IEEE, p 450-455

40. Zhang X, Jin J, Meng H, Wang Z (2011) A sensing optimal proposal based on intelligent street lighting system. In: Communication Technology and Application (ICCTA 2011), IET International Conference on. IET, p 968-971

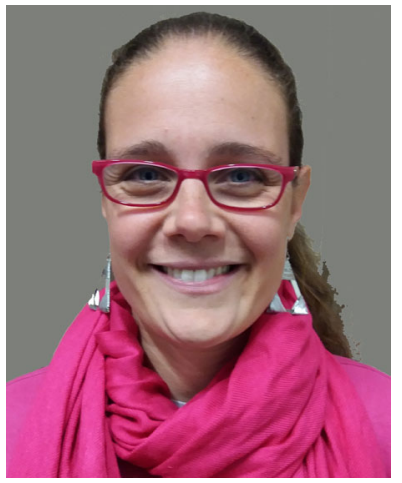

Amalia Luque received her Industrial Engineering degree in 2007, Master's in Automation, Robotics and Telematics in 2010 and an Industrial Engineering Doctoral Degree in 2014. She has been involved in teaching related to Project Engineering at the University of Seville since 2015, where she is currently an Assistant Professor. Her main areas of research are control, business intelligence, data mining, feature extraction and artificial intelligence.

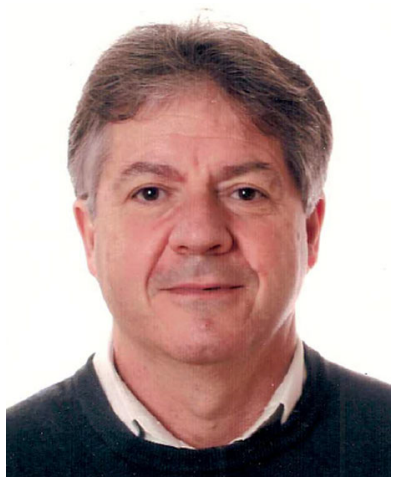

Francisco Aguayo received his Industrial Engineering degree in 1990 and an Industrial Engineering Doctoral Degree in 2002. He has been involved in teaching with the Project Engineering at the University of Seville since 1982, where he is currently an Associate Professor. His main areas of research are industrial ergonomics and environmental quality, neuro-ergonomics, cognitive ergonomics, cognitive interaction and interface design of products and systems. 


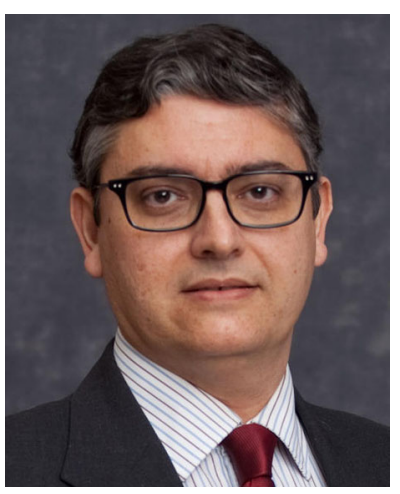

Juan Ramón Lama received his Electronic Engineering degree in 1992 and an Industrial Engineering Doctoral Degree in 2016. He has been involved in related to the Project Engineering at the University of Seville since 1996, where he is currently an Associate Professor. His main areas of research are advanced manufacturing systems, intelligent systems for quality management in extended enterprise environments and, more recently, sustainable industrial systems. 